\title{
On Littlewood-Paley functions and singular integrals
}

\author{
Yong Ding, Dashan FAN and Yibiao PAN
}

(Received August 13, 1999; Revised December 6, 1999)

\begin{abstract}
In this paper, we obtain certain sufficient conditions for the $L^{p}$ boundedness on Littlewood-Paley functions and on some singular integrals. As applications, we study Marcinkiewicz integrals and singular integrals whose kernels are rough not only on the sphere, but also in the radial direction.
\end{abstract}

Key words: Littlewood-Paley theory, $g$-function, singular integral.

\section{Introduction}

Let $\mathbb{R}^{n}$ be $n$-dimensional Euclidean space and $\mathbb{T}^{n}$ be the $n$-dimensional torus. $\mathbb{T}^{n}$ can be identified with $\mathbb{R}^{n} / \Lambda$, where $\Lambda$ is the unit lattice which is the additive group of points in $\mathbb{R}^{n}$ having integral coordinates. For an $L^{1}\left(\mathbb{R}^{n}\right)$ function $\Phi$ we define $\Phi_{t}(x)=2^{-t n} \Phi\left(x / 2^{t}\right), t \in \mathbb{R}$. Then the Fourier transform of $\Phi_{t}$ is $\widehat{\Phi}_{t}(\xi)=\widehat{\Phi}\left(2^{t} \xi\right)$. The Littlewood-Paley $g$-function $g_{\Phi}(f)$ on $\mathbb{R}^{n}$ is defined by

$$
g_{\Phi} f(x)=\left(\int_{\mathbb{R}}\left|\Phi_{t} * f(x)\right|^{2} d t\right)^{1 / 2},
$$

initially, for $f$ in the Schwartz space $\mathcal{S}\left(\mathbb{R}^{n}\right)$.

The Littlewood-Paley $g$-function on $\mathbb{T}^{n}$ can be defined similarly. For $\tilde{f} \in C^{\infty}\left(\mathbb{T}^{n}\right), \tilde{f}$ has the Fourier series

$$
\widetilde{f}(x)=\sum_{k \in \Lambda} a_{k} e^{2 \pi i\langle k, x\rangle} .
$$

We let

$$
G_{\Phi} \tilde{f}(x)=\left(\int_{\mathbb{R}}\left|\widetilde{\Phi}_{t} * \tilde{f}(x)\right|^{2} d t\right)^{1 / 2}
$$

where

$$
\widetilde{\Phi}_{t} * \widetilde{f}(x)=\sum_{k \in \Lambda} \widehat{\Phi}\left(2^{t} k\right) a_{k} e^{2 \pi i\langle k, x\rangle}
$$

1991 Mathematics Subject Classification : 42B20, 42B25. 
The theory of Littlewood-Paley functions has been an important part of harmonic analysis, dating back as far as the early 30's. Readers are refered to [St1] [St2] [St3] for its history and significance. One of well-known results is the following:

Theorem A Suppose that $\Phi \in \mathcal{S}\left(\mathbb{R}^{n}\right)$ satisfies $\int_{\mathbb{R}^{n}} \Phi(x) d x=0$. Then for any $p \in(1, \infty)$

$$
\begin{aligned}
& \left\|g_{\Phi}(f)\right\|_{L^{p}\left(\mathbb{R}^{n}\right)} \leq A\|f\|_{L^{p}\left(\mathbb{R}^{n}\right)}, \\
& \left\|G_{\Phi}(\widetilde{f})\right\|_{L^{p}\left(\mathbb{T}^{n}\right)} \leq A\|\widetilde{f}\|_{L^{p}\left(\mathbb{T}^{n}\right)} .
\end{aligned}
$$

In additional, if $\Phi$ is radial and nonzero, then

$$
\begin{aligned}
& \|f\|_{L^{p}\left(\mathbb{R}^{n}\right)} \leq B\left\|g_{\Phi}(f)\right\|_{L^{p}\left(\mathbb{R}^{n}\right)}, \\
& \|\widetilde{f}\|_{L^{p}\left(\mathbb{T}^{n}\right)} \leq B\left\|G_{\Phi}(\tilde{f})\right\|_{L^{p}\left(\mathbb{T}^{n}\right)},
\end{aligned}
$$

where $A$ and $B$ are positive constants independent of $f$ and $\tilde{f}$.

The proof of (1.2) and $\left(1.2^{\prime}\right)$ can be found in [Fe1]. The proof of (1.3) and $\left(1.3^{\prime}\right)$ is essentially the same as that in [Fe1].

The main purpose of this paper is to lift the smoothness condition on $\Phi$ in Theorem A, and replace it with much weaker conditions. One of our results is the following

Theorem 1 Suppose that $\Phi \in L^{1}\left(\mathbb{R}^{n}\right)$ satisfies

(i) $\left\|\sup _{t \in \mathbb{R}}\left|\Phi_{t}\right| * f\right\|_{L^{q}\left(\mathbb{R}^{n}\right)} \leq C_{q}\|f\|_{L^{q}\left(\mathbb{R}^{n}\right)}$ for all $q>p_{0}$ for some $p_{0} \in(1, \infty)$,

(ii) there are $\alpha, \beta>0, d \in\{1,2, \ldots, n\}$ such that

$$
|\widehat{\Phi}(\xi)| \leq C \min \left\{\left|\Pi_{d} \xi\right|^{\alpha},\left|\Pi_{d} \xi\right|^{-\beta}\right\}
$$

for all $\xi=\left(\xi_{1}, \ldots, \xi_{n}\right) \in \mathbb{R}^{n}$, where $\Pi_{d} \xi=\left(\xi_{1}, \ldots, \xi_{d}\right)$.

Then for every $p, 2 p_{0}\left(p_{0}+1\right)^{-1}<p<2 p_{0}\left(p_{0}-1\right)^{-1}$, there exists $C=$ $C(p)>0$ such that

$$
\left\|g_{\Phi}(f)\right\|_{L^{p}\left(\mathbb{R}^{n}\right)} \leq C\|f\|_{L^{p}\left(\mathbb{R}^{n}\right)}
$$

and

$$
\left\|G_{\Phi}(\tilde{f})\right\|_{L^{p}\left(\mathbb{T}^{n}\right)} \leq C\|\tilde{f}\|_{L^{p}\left(\mathbb{T}^{n}\right)}, \quad 1<p<\infty
$$


for $f \in \mathcal{S}\left(\mathbb{R}^{n}\right)$ and $\tilde{f} \in C^{\infty}\left(\mathbb{T}^{n}\right)$.

When $\Phi \in \mathcal{S}\left(\mathbb{R}^{n}\right)$ and $\int_{\mathbb{R}^{n}} \Phi(x) d x=0$, one sees easily that (i) is satisfied and $|\widehat{\Phi}(\xi)| \leq C \min \left\{|\xi|,|\xi|^{-1}\right\}$ always holds.

We also define the operators $T_{\Phi}$ and $\mathcal{T}_{\Phi}$ on $\mathcal{S}\left(\mathbb{R}^{n}\right)$ and $C^{\infty}\left(\mathbb{T}^{n}\right)$, respectively, by

$$
\begin{aligned}
& T_{\Phi} f(x)=\int_{\mathbb{R}} \Phi_{t} * f(x) d t, \\
& \mathcal{T}_{\Phi} \widetilde{f}(x)=\int_{\mathbb{R}} \widetilde{\Phi}_{t} * \widetilde{f}(x) d t .
\end{aligned}
$$

Theorem 2 If $\Phi$ satisfies the conditions in Theorem 1, then for every $p \in\left(2 p_{0}\left(p_{0}+1\right)^{-1}, 2 p_{0}\left(p_{0}-1\right)^{-1}\right)$,

$$
\begin{aligned}
\left\|T_{\Phi} f\right\|_{L^{p}\left(\mathbb{R}^{n}\right)} & \leq C\|f\|_{L^{p}\left(\mathbb{R}^{n}\right)}, \\
\left\|\mathcal{T}_{\Phi} f\right\|_{L^{p}\left(\mathbb{T}^{n}\right)} & \leq C\|f\|_{L^{p}\left(\mathbb{T}^{n}\right)} .
\end{aligned}
$$

Theorems 1 and 2 will be proved in the second section. As applications of Theorem 1, we will study the Marcinkiewicz integrals (of one dimension) in Section 3 and (of $n$ dimension) in Section 4. As an application of Theorem 2, in Section 5 we will reprove the $L^{p}$-boundedness for certain well-known singular integrals. Recently, by using the extrapolation theorem of Rubio de Francia, S. Sato $[S]$ obtained Theorem 1 under a different condition from (i).

Throughout this paper, the letter $C$ will denote a positive constant that may vary at each occurrence but is independent of the essential variables. We also denote $f(x) \cong g(x)$ if there exist positive constants $A$ and $B$ independent of $x$ such that $A f(x) \leq g(x) \leq B f(x)$.

\section{Proofs of Theorems 1 and 2}

\subsection{Proof of Theorem 1}

The proof of (1.5) is essentially the same as that of (1.4). The only non-trivial part is to prove that the condition

$$
\left\|\sup _{t \in \mathbb{R}}\left|\Phi_{t}\right| * f\right\|_{L^{p}\left(\mathbb{R}^{n}\right)} \leq C\|f\|_{L^{p}\left(\mathbb{R}^{n}\right)}
$$


implies

$$
\left\|\sup _{t \in \mathbb{R}}\left|\phi_{t} * \widetilde{f}\right|\right\|_{L^{p}\left(\mathbb{T}^{n}\right)} \leq C\|\widetilde{f}\|_{L^{p}\left(\mathbb{T}^{n}\right)}
$$

where $\phi_{t}(x)=\sum_{k \in \Lambda} 2^{-t n}\left|\Phi\left(2^{-t}(x+2 \pi k)\right)\right|$.

But this fact was proved by Kenig and Tomas in [KT]. For this reason, we will prove (1.4) only. Take a radial function $\Psi \in \mathcal{S}\left(\mathbb{R}^{d}\right)$ such that the values of its Fourier transform $\widehat{\Psi}$ are between 0 and 1 and it satisfies

$$
\begin{aligned}
& \int_{\mathbb{R}} \widehat{\Psi}\left(2^{s}\right) d s=1, \\
& \operatorname{supp}(\widehat{\Psi}) \subseteq\left\{y \in \mathbb{R}^{d}: 2^{-1}<|y| \leq 2\right\} .
\end{aligned}
$$

Let $\delta$ be the Dirac delta function on $\mathbb{R}^{n-d}$. Then by checking the Fourier transforms, it is easy to see that for any test function $f \in \mathcal{S}\left(\mathbb{R}^{n}\right)$,

$$
f \cong \int_{\mathbb{R}}\left(\Psi_{s} \otimes \delta\right) * f d s
$$

Also, by Theorem A, it is easy to see that

$$
\left\|\left(\int_{\mathbb{R}}\left|\left(\Psi_{s} \otimes \delta\right) * f(\cdot)\right|^{2} d s\right)^{1 / 2}\right\|_{L^{p}\left(\mathbb{R}^{n}\right)} \leq C\|f\|_{L^{p}\left(\mathbb{R}^{n}\right)} .
$$

By (2.1) and Minkowski inequality, we have that

$$
\begin{aligned}
g_{\Phi} f(x) & \cong\left(\int_{\mathbb{R}}\left(\int_{\mathbb{R}}\left(\Psi_{s+t} \otimes \delta\right) * \Phi_{t} * f(x) d s\right)^{2} d t\right)^{1 / 2} \\
& \leq \int_{\mathbb{R}} I_{s} f(x) d s
\end{aligned}
$$

where

$$
I_{s} f(x)=\left(\int_{\mathbb{R}}\left|\left(\Psi_{s+t} \otimes \delta\right) * \Phi_{t} * f(x)\right|^{2} d t\right)^{1 / 2} .
$$

By Minkowski inequality again, for any $p \in(1, \infty)$

$$
\left\|g_{\Phi} f\right\|_{L^{p}\left(\mathbb{R}^{n}\right)} \leq C \int_{\mathbb{R}}\left\|I_{s} f\right\|_{L^{p}\left(\mathbb{R}^{n}\right)} d s .
$$

Now we prove that there exists a constant $C=C_{p}$ independent of $s$ such that for $p \in\left(2 p_{0}\left(p_{0}+1\right)^{-1}, 2 p_{0}\left(p_{0}-1\right)^{-1}\right)$,

$$
\left\|I_{s} f\right\|_{L^{p}\left(\mathbb{R}^{n}\right)} \leq C\|f\|_{L^{p}\left(\mathbb{R}^{n}\right)} .
$$


We define a linear operator $T$ on any function $\mathcal{F}(x, t)$ by $T \mathcal{F}(x, t)=\Phi_{t} *$ $\mathcal{F}(x, t)$ and want to prove the mixed norm inequality

$$
\|\| T \mathcal{F}\left\|_{L^{2}(\mathbb{R})}\right\|_{L^{p}\left(\mathbb{R}^{n}\right)} \leq C\|\| \mathcal{F}\left\|_{L^{2}(\mathbb{R})}\right\|_{L^{p}\left(\mathbb{R}^{n}\right)} .
$$

From the definition, it is easy to see that

$$
\|\| T \mathcal{F}\left\|_{L^{1}(\mathbb{R})}\right\|_{L^{1}\left(\mathbb{R}^{n}\right)} \leq C_{1}\|\| \mathcal{F}\left\|_{L^{1}(\mathbb{R})}\right\|_{L^{1}\left(\mathbb{R}^{n}\right)} .
$$

By (i), we have

$$
\|\| T \mathcal{F}\left\|_{L^{\infty}(\mathbb{R})}\right\|_{L^{q}\left(\mathbb{R}^{n}\right)} \leq C_{2}\|\| \mathcal{F}\left\|_{L^{\infty}(\mathbb{R})}\right\|_{L^{q}\left(\mathbb{R}^{n}\right)}
$$

for any $q>p_{0}$. Clearly the above constants $C_{1}$ and $C_{2}$ are independent of the essential variables. Thus by interpolation and duality we obtain (2.4) for all $\left.p \in\left(2 p_{0}\left(p_{0}+1\right)^{-1}\right), 2 p_{0}\left(p_{0}-1\right)^{-1}\right)$. In particular, letting $\mathcal{F}(x, t)=$ $\left(\Psi_{s+t} \otimes \delta\right) * f(x)$, then we obtain

$$
\begin{aligned}
\left\|I_{s} f\right\|_{L^{p}\left(\mathbb{R}^{n}\right)} & =\|\| T \mathcal{F}\left\|_{L^{2}(\mathbb{R})}\right\|_{L^{p}\left(\mathbb{R}^{n}\right)} \leq C\|\| \mathcal{F}\left\|_{L^{2}(\mathbb{R})}\right\|_{L^{p}\left(\mathbb{R}^{n}\right)} \\
& =C\left\|g_{\Psi}(f)\right\|_{L^{p}\left(\mathbb{R}^{n}\right)} \leq C\|f\|_{L^{p}\left(\mathbb{R}^{n}\right)} .
\end{aligned}
$$

(2.3) is proved.

If $s>0$, by Plancherel's theorem

$$
\left\|I_{s} f\right\|_{2}^{2} \leq C \int_{\mathbb{R}} \int_{E_{t, s} \times \mathbb{R}^{n-d}}\left|\widehat{\Phi}\left(2^{t} \xi\right)\right|^{2}|\widehat{f}(\xi)|^{2} d \xi d t
$$

where

$$
E_{t, s}=\left\{\eta \in \mathbb{R}^{d}: 2^{-t-s-1} \leq|\eta|<2^{-t-s+1}\right\} .
$$

Thus by (ii), we know that if $\Pi_{d} \xi \in E_{t, s}$ then

$$
\left|\widehat{\Phi}\left(2^{t} \xi\right)\right| \leq C\left|2^{t} \Pi_{d} \xi\right|^{\alpha} \leq C 2^{-\alpha s} .
$$

Thus

$$
\left\|I_{s} f\right\|_{2}^{2} \leq C 2^{-2 \alpha s} \int_{\mathbb{R}} \int_{E_{t, s} \times \mathbb{R}^{n-d}}|\widehat{f}(\xi)|^{2} d \xi d t .
$$

By the definition of $E_{t, s}$, it is easy to see that

$$
\left\|I_{s} f\right\|_{2}^{2} \leq C 2^{-2 \alpha s}\|\widehat{f}\|_{2}^{2} \cong C 2^{-2 \alpha s}\|f\|_{2}^{2},
$$


which shows that

$$
\left\|I_{s}\right\|_{L^{2} \rightarrow L^{2}} \leq C 2^{-\alpha s} .
$$

Noting $2 \in\left(2 p_{0}\left(p_{0}+1\right)^{-1}, 2 p_{0}\left(p_{0}-1\right)^{-1}\right)$, we now use interpolation between (2.3) and (2.5) to obtain a $\theta>0$ such that

$$
\left\|I_{s} f\right\|_{p} \leq C 2^{-s \theta}\|f\|_{p}
$$

Thus we have, for all $p \in\left(2 p_{0}\left(p_{0}+1\right)^{-1}, 2 p_{0}\left(p_{0}-1\right)^{-1}\right)$,

$$
\int_{0}^{\infty}\left\|I_{s} f\right\|_{p} d s \leq C\|f\|_{p}
$$

If $s<0$, we have

$$
I_{s} f=\int_{\mathbb{R}} \mid\left(\left.\Phi_{t} *\left(\Psi_{s+t} \otimes \delta\right) * f\right|^{2} d t\right)^{1 / 2} .
$$

Thus

$$
\left\|I_{s} f\right\|_{2}^{2} \leq \int_{\mathbb{R}} \int_{E_{t, s} \times \mathbb{R}^{n-d}}\left|\widehat{\Phi}_{t}(\xi)\right|^{2}|\widehat{f}(\xi)|^{2} d \xi d t .
$$

Similar to (2.5), by (ii), it is easy to see

$$
\left\|I_{s}\right\|_{L^{2} \rightarrow L^{2}} \leq C 2^{\beta s} \text {. }
$$

We now use interpolation to obtain that

$$
\left\|I_{s} f\right\|_{p} \leq C 2^{s \theta}\|f\|_{p}
$$

for some $\theta>0$. This shows that, for all $p \in\left(2 p_{0}\left(p_{0}+1\right)^{-1}, 2 p_{0}\left(p_{0}-1\right)^{-1}\right)$,

$$
\int_{-\infty}^{0}\left\|I_{s} f\right\|_{p} d s \leq C\|f\|_{p} .
$$

Clearly, the constant $C$ above is independent of the essential variables. Theorem 1 is proved.

\subsection{Proof of Theorem 2}

Similar to the proof of Theorem 1, we prove (1.8) only. Choose the function $\Psi$ as in the proof of Theorem 1. Then by $\left(1.2^{\prime}\right)$

$$
\left\|T_{\Phi} f\right\|_{L^{p}\left(\mathbb{R}^{n}\right)} \leq C\left\|g_{\Psi}\left(T_{\Phi}(f)\right)\right\|_{L^{p}\left(\mathbb{R}^{n}\right)}
$$


where

$$
\begin{aligned}
g_{\Psi}\left(T_{\Phi} f\right)(x) & =\left(\int_{\mathbb{R}}\left|\int_{\mathbb{R}} \Psi_{s} * \Phi_{t+s} * f(x) d t\right|^{2} d s\right)^{1 / 2} \\
& \leq \int_{\mathbb{R}}\left(\int_{\mathbb{R}}\left|\Psi_{s} * \Phi_{t+s} * f(x)\right|^{2} d s\right)^{1 / 2} d t .
\end{aligned}
$$

Therefore, using the same argument as that in Theorem 1, we obtain Theorem 2 .

Theorem 1 and Theorem 2 has the following corollary.

Corollary Let $m, n \in \mathbb{N}$ and $A: \mathbb{R}^{n} \rightarrow \mathbb{R}^{m}$ be a linear transformation. Suppose that $\Phi \in L^{1}\left(\mathbb{R}^{n}\right)$ satisfies

(i) $\quad\left\|\sup _{t \in \mathbb{R}}\left|\Phi_{t}\right| * f\right\|_{L^{q}\left(\mathbb{R}^{n}\right)} \leq C_{1}\|f\|_{L^{q}\left(\mathbb{R}^{n}\right)}$ for $q>p_{0}$,

(ii') $|\widehat{\Phi}(\xi)| \leq C_{2} \min \left\{|A \xi|^{\alpha},|A \xi|^{-\beta}\right\}$

for some $\alpha, \beta>0$ and all $\xi \in \mathbb{R}^{n}$. Then (1.4) and (1.8) remain true with a constant $C$ independent of the linear transformation $A$.

Proof. Let $d=\operatorname{rank}(A)$. Then there are nonsingular linear transformations $G: \mathbb{R}^{n} \rightarrow \mathbb{R}^{n}$ and $H: \mathbb{R}^{d} \rightarrow \mathbb{R}^{d}$ such that $\left|H \Pi_{d} G \xi\right| \leq|A \xi| \leq$ $m\left|H \Pi_{d} G \xi\right|$ for all $\xi \in \mathbb{R}^{n}$. Let $U=G^{-1} \circ\left(H^{-1} \otimes \operatorname{id}_{\mathbb{R}^{n-d}}\right)$ and

$$
\Psi(x)=\Phi\left(\left(U^{t}\right)^{-1} x\right)|\operatorname{det} U|^{-1} .
$$

Then by $\widehat{\Psi}(\xi)=\widehat{\Phi}(U \xi)$, we obtain $|\widehat{\Psi}(\xi)| \leq C_{3} \min \left\{\left|\Pi_{d} \xi\right|^{\alpha},\left|\Pi_{d} \xi\right|^{-\beta}\right\}$.

It is easy to see that (i) in Theorem 1 remains valid when $\Phi$ is replaced by $\Psi$. By letting $F(x)=f\left(\left(U^{t}\right)^{-1} x\right)$, we obtain

$$
\begin{aligned}
\left\|T_{\Phi} f\right\|_{L^{p}\left(\mathbb{R}^{n}\right)}^{p} & \leq \int_{\mathbb{R}^{n}}\left|T_{\Psi} F\left(U^{t} x\right)\right|^{p} d x \\
& \leq C_{p} \int_{\mathbb{R}^{n}}|F(y)|^{p} d y|\operatorname{det}(U)|^{-1}=C_{p}\|f\|_{L^{p}\left(\mathbb{R}^{n}\right)}^{p}
\end{aligned}
$$

which proves (1.8). A similar argument can be used to obtain (1.4).

\section{One dimensional Marcinkiewicz functions}

In [M], in order to study boundary values of analytic functions, Marcinkiewicz introduced the function $\mu(\widetilde{f})$ on the one-dimensional 
torus $\mathbb{T}$ :

$$
\mu(\tilde{f})(x)=\left(\int_{0}^{2 \pi}|F(x+t)+F(x-t)-2 F(x)|^{2} t^{-3} d t\right)^{1 / 2}
$$

where $F(x)=\int_{0}^{x} \widetilde{f}(s) d s$. Marcinkiewicz conjectured, and Zygmund proved [Z], that

$$
\|\mu(\tilde{f})\|_{L^{p}(\mathbb{T})} \leq C\|\tilde{f}\|_{L^{p}(\mathbb{T})}, \quad 1<p<\infty .
$$

An analog of Marcinkiewicz function on $\mathbb{R}$ is defined by

$$
\nu(f)=\left(\int_{0}^{\infty}|F(x+t)+F(x-t)-2 F(x)|^{2} t^{-3} d t\right)^{1 / 2}
$$

where $F(x)=\int_{0}^{x} f(s) d s$. Waterman [W] extended Zygmund's method, without essential change, to prove that

$$
\|\nu(f)\|_{L^{p}(\mathbb{R})} \leq C\|f\|_{L^{p}(\mathbb{R})}
$$

The arguments in either case, however, used the complex function theory such as Blaschke product decompositions of analytic functions, are far from simple. Recall $F(x+t)+F(x-t)-2 F(x)=\int_{x}^{x+t} f(s) d s-\int_{x-t}^{x} f(s) d s$. Then it is easy to see that $\nu(f)(x) \cong g_{\Phi}(f)(x)$ and $\mu(\widetilde{f})(x) \cong G_{\Phi} \widetilde{f}(x)$ where $\Phi(x)=\operatorname{sign} x$ if $x \in[-1,1]$ and $\Phi(x)=0$ if $x \notin[-1,1]$. Also it is easy to check that $\Phi$ satisfies all conditions in Theorem 1. So using Theorem 1 we reprove (3.2) and (3.4). Moreover, we can extend the Marcinkiewicz function to more general functions $\left(g_{\lambda} f\right)(x)$, where $\lambda(x)=\lambda(x, a, b)=$ $\operatorname{sign} x|x|^{a}(1-|x|)^{b}$ if $x \in(-1,1)$, and $\lambda(x)=0$ if $x \notin(-1,1)$ with $a, b>-1$. Clearly $g_{\lambda} f(x) \cong \nu(f)(x)$ and $G_{\lambda} \widetilde{f}(x) \cong \mu(\widetilde{f})(x)$ if $a=b=0$, we have the following theorem

Theorem 3 Let $\gamma=\min \{a, b\}$. Then we have

$$
\begin{gathered}
\left\|g_{\lambda} f\right\|_{L^{p}(\mathbb{R})} \leq C\|f\|_{L^{p}(\mathbb{R})} \\
\left\|G_{\lambda} \widetilde{f}\right\|_{L^{p}(\mathbb{T})} \leq C\|\tilde{f}\|_{L^{p}(\mathbb{T})}
\end{gathered}
$$

for all $1<p<\infty$ if $\gamma \geq 0$; and for all $p \in(2 /(2+\gamma),-2 / \gamma)$ if $-1<\gamma<0$.

Proof. The proof for the case $\gamma \geq 0$ is easy, we prove the case $\gamma \in(-1,0)$ only. To this end, it suffices to check that $\lambda(x)$ satisfies (i) and (ii) in Theorem 1. For fixed $r^{\prime}$ such that $r \gamma>-1$, by Hölder's inequality, for any 
$f(x) \geq 0$

$$
\begin{aligned}
\left|\lambda_{t}\right| * f(x) & \leq C 2^{-t} \int_{-2^{t}}^{2^{t}}\left(|s| 2^{-t}\right)^{a}\left(1-|s| 2^{-t}\right)^{b} f(x-s) d s \\
& \leq C\left(2^{-t} \int_{-2^{t}}^{2^{t}}|f(x-s)|^{r^{\prime}} d s\right)^{1 / r^{\prime}} \leq C\left(M\left(f^{r^{\prime}}\right)(x)\right)^{1 / r^{\prime}}
\end{aligned}
$$

where $C$ is independent of $t$, and $M f$ is the one-dimensional HardyLittlewood maximal function. This shows that if $q>r^{\prime}$ then $\| \sup _{t \in \mathbb{R}}\left|\lambda_{t}\right| *$ $f\left\|_{L^{q}(\mathbb{R})} \leq C\right\| f \|_{L^{q}(\mathbb{R})}$. So $\lambda(x)$ satisfies (i) in Theorem 1 for $p_{0}=r^{\prime}$. On the other hand, that $\lambda(x)$ is an odd function implies that

$$
\widehat{\lambda}(\xi) \cong \int_{0}^{1} s^{a}(1-s)^{b} \sin s \xi d s
$$

and $|\widehat{\lambda}(\xi)| \leq C|\xi|$ if $|\xi| \leq 1$. If $|\xi|>1$, without loss of generality, we assume $|\xi|>4$ and let

$$
\begin{aligned}
|\widehat{\lambda}(\xi)| \leq & \left|\int_{1 / 2}^{1-|\xi|^{-1}} s^{a}(1-s)^{b} \sin s \xi d s\right|+C \int_{1-|\xi|^{-1}}^{1}(1-s)^{b} d s \\
& +C\left|\int_{|\xi|^{-1}}^{1 / 2} s^{a}(1-s)^{b} \sin s \xi d s\right|+C \int_{0}^{|\xi|^{-1}} s^{a} d s
\end{aligned}
$$

Then an easy computation shows that $|\widehat{\lambda}(\xi)| \leq C|\xi|^{-1-\gamma}$. Noting $1+\lambda=$ $\beta>0$, we prove that $\lambda(x)$ also satisfies (ii) in Theorem 1. Noting that $p_{0}=r^{\prime}>(1+\gamma)^{-1}$, by Theorem 1 we obtain Theorem 3 .

Some similar version of Theorem 3 was also obtained by Sunouchi [S] and by Sato [Sa2]. In particular, recently Sato [Sa2] used a different proof to obtain some better estimates for $g_{\lambda} f$ in the cases $-1<b<0$ and $a=0,1$, due to the elegance of his proof (see Theorem 4 in [Sa2]).

\section{Marcinkiewicz function on $\mathbb{R}^{n}, n \geq 2$}

Let $\Omega(x)$ be a function which is homogeneous of degree 0 , and which satisfies

$$
\int_{S^{n-1}} \Omega(x) d \sigma(x)=0
$$


where $S^{n-1}$ is the unit sphere on $\mathbb{R}^{n}, n \geq 2$ and $d \sigma(x)$ is the normalized Lebesque measure of $S^{n-1}$. In [St1], Stein defined the function $F_{t}(x)$ by $F_{t}(x)=\int_{|y|<t} f(x-y) \Omega\left(y^{\prime}\right)|y|^{-n+1} d y$ where $y^{\prime}=y /|y|$. It was noted that when $n=1, \Omega\left(y^{\prime}\right)=\operatorname{sign} y$, so that $F_{t}(x)=F(x+t)+F(x-t)-2 F(x)$. Stein defined the Marcinkiewicz function on $\mathbb{R}^{n}$ by

$$
\nu_{\Omega}(f)=\left(\int_{0}^{\infty}\left|F_{t}(x)\right|^{2} t^{-3} d t\right)^{1 / 2}
$$

and proved, by using the "real method", that

$$
\left\|\nu_{\Omega}(f)\right\|_{L^{p}\left(\mathbb{R}^{n}\right)} \leq C\|f\|_{L^{p}\left(\mathbb{R}^{n}\right)}
$$

provided that $\Omega$ satisfies a Lipschitz condition of order $\alpha, 0<\alpha \leq 1$. Let $\Phi(x)=\chi_{B}(x)|x|^{-n+1} \Omega(x)$ where $\chi_{B}$ is the characteristic function of the unit ball $B=\{|x|<1\}$. It is easy to see that $\nu_{\Omega} f \cong g_{\Phi} f$ and that $\Phi$ satisfies all conditions on Theorem 1. So by Theorem 1, we reprove (4.1). Actually, the smoothness condition on $\Phi$ in Stein's theorem can be replaced by certain roughness condition (see following Theorem 4).

For a measurable function $b(t)$ on $\mathbb{R}_{+}$, we say that $b \in \Delta_{\varepsilon}, \varepsilon \geq 1$, if

$$
\|b\|_{\Delta_{\varepsilon}}=\sup _{R>0} R^{-1} \int_{R / 2}^{R}|b(t)|^{\varepsilon} d t<\infty .
$$

Theorem 4 Let $\Phi(x)=\tau(|x|) \Omega\left(x^{\prime}\right)$. Suppose that $\Omega \in L^{q}\left(S^{n-1}\right), q>$ 1 satisfies (4.1), and that $|\tau(s)| \leq C b(s) s^{-n+\rho}$ if $0 \leq s \leq 1,|\tau(s)| \leq$ $C b(s) s^{-n-\rho}$ if $s \geq 1$, for some $\rho \in(0, n)$ and $b \in \Delta_{\varepsilon}$ for some $\varepsilon>1$. Then $\left\|g_{\Phi} f\right\|_{L^{p}\left(\mathbb{R}^{n}\right)} \leq C\|f\|_{L^{p}\left(\mathbb{R}^{n}\right)}$ and $\left\|G_{\Phi} f\right\|_{L^{p}\left(\mathbb{T}^{n}\right)} \leq C\|f\|_{L^{p}\left(\mathbb{T}^{n}\right)}$.

Proof. By Theorem 1, we need to check that the function $\Phi$ satisfies (i) and (ii) in Theorem 1. For $f(x) \geq 0$

$$
\begin{aligned}
\left|\Phi_{t}\right| * f(x) \leq & C 2^{-t \rho} \int_{|y|<2^{t}} b\left(2^{-t}|y|\right)|y|^{-n+\rho}\left|\Omega\left(y^{\prime}\right)\right| f(x-y) d y \\
& +C 2^{t \rho} \int_{|y| \geq 2^{t}} b\left(2^{-t}|y|\right)|y|^{-n-\rho}\left|\Omega\left(y^{\prime}\right)\right| f(x-y) d y \\
= & \Phi_{t}^{1} f(x)+\Phi_{t}^{2} f(x) .
\end{aligned}
$$


For fixed $t>0$, let $\zeta$ be an integer such such that $t \leq \zeta<t+1$. Then

$$
\Phi_{t}^{1} f(x) \leq C 2^{-t \rho} \sum_{j=-\infty}^{\zeta} 2^{j \rho} \sigma^{*} f(x) \leq C \sigma^{*} f(x)
$$

where the constant $C$ is independent of $t$ and

$$
\sigma^{*} f(x)=\sup _{j \in \mathbb{Z}} 2^{-n j} \int_{2^{j-1} \leq|y|<2^{j}} b\left(2^{-t}|y|\right)\left|\Omega\left(y^{\prime}\right)\right| f(x-y) d y .
$$

Let $B(s)=b\left(2^{-t} s\right)$. It is easy to check that $\|B\|_{\Delta_{\varepsilon}}=\|b\|_{\Delta_{\varepsilon}}$. So by Theorem 8.1 in [FP1], we know that $\left\|\sigma^{*} f\right\|_{L^{p}\left(\mathbb{R}^{n}\right)} \leq C\|f\|_{L^{p}\left(\mathbb{R}^{n}\right)}$, which shows that

$$
\left\|\sup _{t \in \mathbb{R}} \Phi_{t}^{1} f\right\|_{L^{p}\left(\mathbb{R}^{n}\right)} \leq C\|f\|_{L^{p}\left(\mathbb{R}^{n}\right)}
$$

Using the same argument, we have

$$
\Phi_{t}^{2} f(x) \leq C 2^{t \rho} \sum_{j=\zeta-1}^{\infty} 2^{-t \rho} \sigma^{*} f(x) \leq C \sigma^{*} f(x)
$$

where $C$ is independent of $t$. This shows that

$$
\left\|\sup _{t \in \mathbb{R}} \Phi_{t}^{2} f\right\|_{L^{p}\left(\mathbb{R}^{n}\right)} \leq C\|f\|_{L^{p}\left(\mathbb{R}^{n}\right)}
$$

By (4.5) and (4.6), we know that $\Phi$ satisfies (i) in Theorem 1 .

Next we check that $\Phi$ satisfies (ii) in Theorem 1. In fact, by the canceliation condition on $\Omega$,

$$
\begin{aligned}
|\widehat{\Phi}(\xi)| \leq & C \int_{1}^{\infty} b(s) s^{-\rho-1}\left|\int_{S^{n-1}} \Omega\left(y^{\prime}\right)\left(e^{-i s\left\langle y^{\prime}, \xi\right\rangle}-1\right) d \sigma\left(y^{\prime}\right)\right| d s \\
& +C \int_{0}^{1} b(s) s^{\rho-1}\left|\int_{S^{n-1}} \Omega\left(y^{\prime}\right)\left(e^{-i s\left\langle y^{\prime}, \xi\right\rangle}-1\right) d \sigma\left(y^{\prime}\right)\right| d s .
\end{aligned}
$$

Note that $\left|\int_{S^{n-1}} \Omega\left(y^{\prime}\right)\left(e^{i s\left\langle y^{\prime}, \xi\right\rangle}-1\right) d \sigma\left(y^{\prime}\right)\right| \leq C \min \{1, s|\xi|\}$. It is easy to see that we can choose a $\alpha>0$ and $\alpha<\rho$ such that

$$
|\widehat{\Phi}(\xi)| \leq C|\xi|^{\alpha} .
$$

For any fix $\xi \neq 0$, we choose a rotation 0 such that $0(\xi)=|\xi| \mathbf{1}=$ 
$|\xi|(1,0, \ldots, 0)$. Let $y^{\prime}=\left(s, y_{2}^{\prime}, y_{3}^{\prime}, \ldots, y_{n}^{\prime}\right)$. Then it is easy to see that

$$
\widehat{\Phi}(\xi)=\int_{0}^{\infty} \tau(u) u^{n-1} \int_{S^{n-1}} \Omega\left(0^{-1}\left(y^{\prime}\right)\right) e^{-i u|\xi|\left\langle 1, y^{\prime}\right\rangle} d \sigma\left(y^{\prime}\right) d u
$$

where $0^{-1}$ is the inverse of 0 . Now $\Omega\left(0^{-1}\left(y^{\prime}\right)\right)$ is again an $L^{q}$ function, so we still write it by $\Omega\left(y^{\prime}\right)$. Thus, using Hölder's inequality and by changing variables, we have

$$
\begin{aligned}
|\widehat{\Phi}(\xi)| \leq C|\xi|^{\rho} \int_{|\xi|}^{\infty} b(u /|\xi|) u^{-\rho-1}|\widehat{h}(u)| d u \\
\quad+C|\xi|^{-\rho} \int_{0}^{|\xi|} b(u /|\xi|) u^{\rho-1}|\widehat{h}(u)| d u \\
=C|\xi|^{\rho} J_{1}(\xi)+C|\xi|^{-\rho} J_{2}(\xi),
\end{aligned}
$$

where $\widehat{h}$ is the Fourier transform of

$$
h(s)=\left(1-s^{2}\right)^{(n-3) / 2} \chi_{(|s|<1)}(s) \int_{S^{n-2}} \Omega\left(s,\left(1-s^{2}\right)^{1 / 2} \widetilde{y}\right) d \sigma(\widetilde{y}) .
$$

$h(s)$ is an $L^{r}(\mathbb{R})$ function for some $r \in(1, q]$ and its $L^{r}$-norm is bounded by the $L^{q}$-norm of $\Omega$ (see page 141 of [LTW]). Since $\|b\|_{\Delta_{\delta}} \leq C\|b\|_{\Delta_{\varepsilon}}$ if $\varepsilon>\delta$, we may assume that $\varepsilon>1$ is sufficiently close to 1 such that $\varepsilon<r$, and $\varepsilon(\rho-1)>-1$. Now by Hölder's and Hausdorff-Young's inequalities,

$$
\begin{aligned}
|\xi|^{\rho} J_{1}(\xi) & \leq|\xi|^{\rho}\left(\int_{|\xi|}^{\infty} b(u /|\xi|)^{\varepsilon} u^{-\varepsilon(\rho+1)} d u\right)^{1 / \varepsilon}\|h\|_{L^{\varepsilon}(\mathbb{R})} \\
& \leq C\|h\|_{L^{r}(\mathbb{R})}\left(|\xi|^{-\varepsilon(\rho+1)+1+\varepsilon \rho} \int_{1}^{\infty} b(u)^{\varepsilon} u^{-\varepsilon(\rho+1)} d u\right)^{1 / \varepsilon} .
\end{aligned}
$$

Thus it is easy to see

$$
|\xi|^{\rho} J_{1}(\xi) \leq C|\xi|^{-1 / \varepsilon^{\prime}}\|b\|_{\Delta_{\varepsilon}}^{1 / \varepsilon}
$$

Similarly,

$$
\begin{aligned}
|\xi|^{-\rho} J_{2}(\xi) & \leq C\left(|\xi|^{-\varepsilon(1-\rho)+1-\varepsilon \rho} \int_{0}^{1} b(u)^{\varepsilon} u^{\varepsilon(\rho-1)} d u\right)^{1 / \varepsilon} \\
& \leq C|\xi|^{-1 / \varepsilon^{\prime}}\|b\|_{\Delta_{\varepsilon}}^{1 / \varepsilon} .
\end{aligned}
$$

By (4.8) and (4.9), we obtain that $\Phi$ satisfies (ii) in Theorem 1 . 


\section{Singular integrals}

Let $\Omega \in L^{1}\left(S^{n-1}\right)$ satisfy (4.1) and $b \in L^{\infty}\left(\mathbb{R}_{+}\right)$. The singular integral with rough kernel is defined by

$$
T f(x)=\text { p.v. } \int_{\mathbb{R}^{n}} b(|y|)|y|^{-n} \Omega\left(y^{\prime}\right) f(x-y) d y .
$$

The study of the operator $T f$ began with Calderón-Zygmund's pioneering papers [CZ1] [CZ2] and subsequently by many other authors (see [Fe1], [DR] [LTW], [FT1], [FP2]). The best result known so far, concerning the size of $\Omega$, is that if $\Omega$ is in the Hardy space $H^{1}\left(S^{n-1}\right)$ then $T$ is bounded on $L^{p}\left(\mathbb{R}^{n}\right)$ (see [FP2] $)$. The Hardy space is defined by

$$
H^{1}\left(S^{n-1}\right)=\left\{\Omega \in L^{1}\left(S^{n-1}\right):\|\Omega\|_{H^{1}}<\infty\right\}
$$

where $\|\Omega\|_{H^{1}}=\left\|\sup _{0 \leq r<1}\left|\int_{S^{n-1}} \Omega\left(y^{\prime}\right) P_{r x^{\prime}}\left(y^{\prime}\right) d \sigma\left(y^{\prime}\right)\right|\right\|_{L^{1}\left(S^{n-1}\right)}$ and $P_{r}$ is the Poisson kernel on $S^{n-1}$. It is well-known that on the sphere, $H^{1}$ contains the space $L \log ^{+} L$ as a proper subspace.

We observe that

$$
T f(x) \cong \int_{\mathbb{R}} \int_{2^{t} \leq|y|<2^{t+1}} B\left(|y| 2^{-t}\right) \Omega\left(y^{\prime}\right)|y|^{-n} f(x-y) d y d t .
$$

where $B(|y|)=b\left(2^{t}|y|\right)$ is again an $L^{\infty}$ function such that $\|B\|_{\infty}=\|b\|_{\infty}$. So let

$$
\omega(y)=B(|y|)|y|^{-n} \Omega\left(y^{\prime}\right) \chi_{\{1<|y| \leq 2\}}(y),
$$

then

$$
T f(x) \cong \int_{\mathbb{R}} \omega_{t} * f(x) d t=T_{\omega} f(x) .
$$

We consider a more general function $\Phi(x)=\Gamma(|x|) \Omega\left(x^{\prime}\right)$, where $\Omega \in$ $L^{1}\left(S^{n-1}\right)$ with $\int \Omega=0,|\Gamma(s)| \leq C s^{-n+\rho}$ if $0 \leq s<1$ and $|\Gamma(s)| \leq C s^{-n-\rho}$ if $s \geq 1$ for some $\rho>0$. Clearly the function $\omega$ is a special case of such $\Phi$. Using Theorem 2, we can reprove the following known result in [FP2].

Theorem 5 If $\Omega \in H^{1}\left(S^{n-1}\right)$ then $T_{\Phi}$ is a bounded operator on $L^{p}\left(\mathbb{R}^{n}\right)$.

Proof. From [C], we know that $\Omega$ has an atomic decomposition

$$
\Omega\left(y^{\prime}\right)=\sum c_{k} a_{k}\left(y^{\prime}\right)
$$


where $\sum\left|c_{k}\right| \leq C\|\Omega\|_{H^{1}\left(S^{n-1}\right)}$ and each $a_{k}$ is an $L^{\infty}$ atom. Recall that an $L^{q}$ atom $a\left(y^{\prime}\right)$ is a function that satisfies

$$
\operatorname{supp}(a) \subseteq B(\zeta, r)
$$

where $B(\zeta, r)=S^{n-1} \cap\left\{y \in \mathbb{R}^{n}:|y-\zeta|<r\right.$ for some $\zeta \in S^{n-1}$ and $2 \geq r>0\}$

$$
\begin{aligned}
& \int_{S^{n-1}} a\left(y^{\prime}\right) d \sigma\left(y^{\prime}\right)=0 \\
& \|a\|_{\infty} \leq r^{(n-1)(1 / q-1)} .
\end{aligned}
$$

So, to prove the theorem, without loss of generality, it suffices to assume that $\Omega$ is an $L^{\infty}$ atom supported in $B(\mathbf{1}, r)$ and to prove that $\left\|T_{\Phi} f\right\|_{L^{p}\left(\mathbb{R}^{n}\right)} \leq$ $C\|f\|_{L^{p}\left(\mathbb{R}^{n}\right)}$ with $C$ independent of $r$. To this end, we check that the function $\Phi$ satisfies (i) and (ii') in Corollary.

Using the same argument as proving Theorem 4, we have

$$
\widehat{\Phi}(\xi)=\int_{0}^{\infty} \Gamma(u) u^{n-1} \int_{S^{n-1}} \Omega\left(0^{-1}\left(y^{\prime}\right)\right) e^{-i u|\xi|\left\langle 1, y^{\prime}\right\rangle} d \sigma\left(y^{\prime}\right) d u,
$$

where $0^{-1}$ is the inverse of 0 . Now $\Omega\left(0^{-1}\left(y^{\prime}\right)\right)$ is again an $\infty$-atom with support in $B(\zeta, r) \cap S^{n-1}$, where $\zeta=\xi^{\prime}$. Thus we have

$$
\widehat{\Phi}(\xi)=\int_{0}^{\infty} \Gamma(u) u^{n-1} \int_{\mathbb{R}} h(s) e^{-i u|\xi| s} d s d u
$$

where $h(s)$ is the function as in (4.7). By Lemma 2.1 in [FP2], we know that $h(s)$ is a $q$-atom on $\mathbb{R}$ with support in $\left(\xi_{1}^{\prime}-A_{r}\left(\xi^{\prime}\right), \xi_{1}^{\prime}+A_{r}\left(\xi^{\prime}\right)\right)$ for some $q \in(1,2)$, where $A_{r}$ is the linear transform $\operatorname{diag}\left[r^{2}, r, \ldots, r\right]$. Thus, by the cancellation condition of $h(s)$ and the the condition on $\Gamma(u)$ we have

$$
\begin{aligned}
|\widehat{\Phi}(\xi)| & \leq \int_{0}^{\infty} u^{n-1} \Gamma(u) \int_{\mathbb{R}} h(s)\left(e^{-i u|\xi| s}-e^{-i u|\xi| \xi_{1}^{\prime}}\right) d s \mid d u \\
& \leq C\left|A_{r} \xi\right|^{\alpha}
\end{aligned}
$$

where $\alpha>0$ and $\alpha<\rho$.

By the same arguments as obtaining (4.8) and (4.9), we have $|\widehat{\Phi}(\xi)| \leq$ $C\left|A_{r} \xi\right|^{1 / q-1}$ for some $q>1$. Thus the function $\Phi$ satisfies (ii').

Checking that $\Phi$ satisfies (i) is exactly the same as those for (4.5) and (4.6), but using Theorem 7.4 of [FP1] instead of Theorem 8.1 in [FP1]. We omit the details. 
A final Remark For an $\Phi \in L^{1}\left(\mathbb{R}^{n}\right)$ the fractional operator $T_{\Phi, \alpha}$ is defined by

$$
T_{\Phi, \alpha} f(x)=\int_{\mathbb{R}} 2^{t \alpha} \Phi_{t} * f(x) d t, \quad \alpha \in \mathbb{R} .
$$

The fractional $g$-functions are defined by

$$
g_{\Phi, \alpha}(f)(x)=\left(\int_{\mathbb{R}}\left|2^{t \alpha} \Phi_{t} * f(x)\right|^{2} d t\right)^{1 / 2} .
$$

We remark that if $\Phi \in \mathcal{S}\left(\mathbb{R}^{n}\right)$ satisfies certain conditions then

$$
\left\|\left(\int_{\mathbb{R}}\left|2^{t \alpha} \Phi_{t} * f(\cdot)\right|^{q} d t\right)^{1 / q}\right\|_{L^{p}\left(\mathbb{R}^{n}\right)}=\|f\|_{\dot{F}_{p}^{\alpha, q}}
$$

is the norm of the Friebel-Lizorkin space $\dot{F}_{p}^{\alpha, q}\left(\mathbb{R}^{n}\right)$ (see $[$ FJW $]$ ).

Acknowledgment The authors would like to express their gratitude to the referee for pointing out a mistake in Theorem 3 in an earlier version of the paper.

\section{References}

[C] Colzani L., Hardy Spaces on Sphere, Ph.D. Thesis. Washington University, St. Louis, MO, 1982.

[CZ1] Calderón A.P. and Zygmund A., On existence of certain singular integrals. Acta. Math. 88 (1952), 85-139.

[CZ2] Calderón A.P. and Zygmund A., On singular integrals. Amer. J. Math. 18 (1956), 289-309.

[DR] Duoandikoetxea J. and Rubio de Francia J.L., Maximal and singular integral operators via Fourier transform estimates. Invent. Math. 84 (1986), 541-561.

[Fe1] Fefferman R., A note on singular integrals. Proc. Amer. Math. Soc. 74 (1979), 266-270.

[Fe2] Fefferman R., Multiparameter Fourier Analysis. Beijing Lectures in Harmonic Analysis, 47-130, Edited by E.M. Stein, Annals of Math. Study \#112, 1986, Princeton Univ. Press.

[FJW] Frazier M., Jawerth B. and Weiss G., Littlewood-Paley Theory and the Study on Function Space. CBMS-AMS Regional Conf., at Auburn Univ., 1989.

[FP1] Fan D. and Pan Y., Singular integral operators with rough kernels supported by subvarieties. Amer. J. Math. 119 (1997), 799-839.

[FP2] Fan D. and Pan Y., A singular integral operator with rough kernel. Proc. Amer. Math. Soc. 125 (1997), 3695-3703. 
[KT] Kenig C. and Tomas P., Maximal operators defined by Fourier multiplier. Studia Math. 68 (1980), 79-83.

[LTW] Lu S., Taibleson M. and Weiss G., Spaces Generated by Blocks. Beijing Normal Univ. Math. Series, 1989, Beijing Normal Univ. Press.

[M] Marcinkiewicz J., Sur quelques integrales de type de Dini. Annales de la Société Polonaise. 17 (1938), 139-154.

[S] Sunouchi G., On the functions of Littlewood-Paley and Marcinkiewicz. Tohoku Math. J. 38 (1984), 505-519.

[Sa1] Sato S., Remarks on square functions in the Littlewood-Paley theory. Bull. Austral. Math. Soc. 58 (1998), 199-211.

[Sa2] Sato S., Multiparameter Marcinkiewicz integrals and a resonance theorem. The Bull. of the Faculty of Education, Kanazawa University 48 (1999).

[St1] Stein E.M., On the functions of Littlewood-Paley, Lusin and Marcinkiewicz. Trans. Amer. Math. Soc. 88 (1958), 430-466.

[St2] Stein E.M., Singular Integrals and Differentiability Properties of Functions. 1970, Princeton Univ. Press.

[St3] Stein E.M., Harmonic Analysis: Real-Variable Methods, Orthogonality And Oscillatory Integrals. Princeton University Press, Princeton, NJ, 1993.

[SW] Stein E.M. and Weiss G., Introduction to Fourier Analysis on Euclidean Spaces. Princeton Univ. Press, Princeton, NJ, 1971.

[W] Waterman D., On an integral of Marcinkiewicz. Proceedings of the International Congress of Mathematicians, Vol.2, 1954, 185-186.

[Z] Zygmund A., On certain integrals. Trans. Amer. Math. Soc. 55 (1944), 170-204.

Yong Ding

Department of Mathematics

Beijing Normal University

Beijing 100875, P.R. China

E-mail: dingy@bnu.edu.cn

Dashan Fan

Department of Mathematics

University of Wisconsin-Milwaukee

Milwaukee, WI 53201, U.S.A.

E-mail: fan@alpha1.csd.uwm.edu

Yibiao Pan

Department of Math. and Statistics

University of Pittsburgh

Pittsburgh, PA 15260, U.S.A.

E-mail: yibiao@tomato.math.pitt.edu 